\title{
Résumé du cadre pancanadien sur les infections transmissibles sexuellement et par le sang
}

\author{
Centre de la lutte contre les maladies transmissibles et les infections ${ }^{1 *}$
}

\section{Résumé}

Les infections transmissibles sexuellement et par le sang (ITSS) restent un problème de santé important au Canada et partout dans le monde. Pour orienter les efforts du Canada et contribuer aux efforts mondiaux visant à réduire les répercussions des ITSS sur la santé, un cadre pancanadien a été élaboré et a été appuyé par les ministres fédéral, provinciaux et territoriaux de la santé. Le cadre présente une approche globale et complète pour lutter contre les ITSS. Étant donné que les ITSS ont en commun des facteurs de risques courants, des voies de transmission et des populations touchées, une approche intégrée a été adoptée. Le cadre établit une vision pour le Canada dans laquelle les ITSS sont rares et où les personnes vivant avec les ITSS reçoivent les soins et le soutien dont elles ont besoin. Le succès du cadre sera mesuré par rapport aux objectifs en matière d'ITSS à l'échelle mondiale et aux progrès réalisés dans l'atteinte des objectifs stratégiques suivants : réduire l'incidence des ITSS au Canada, améliorer l'accès au dépistage, au traitement, aux soins de santé et au soutien continus et réduire la stigmatisation et la discrimination qui créent une vulnérabilité aux ITSS. Le cadre comprend quatre piliers interconnectés qui s'étendent à l'ensemble du suivi des soins des ITSS : la prévention, le dépistage, l'amorce des soins et des traitements, ainsi que les soins et le soutien continus. Le cadre reconnaît que la création d'un environnement favorable englobe I'ensemble de conditions juridiques, sociales, culturelles, physiques et structurelles interreliées qui contribuent au succès des programmes, des politiques et des mesures liées aux ITSS. Grâce à ce cadre, le Canada unifie et communique une approche commune pour lutter contre les ITSS tout en respectant la souplesse exigée par les jurisdictions fédéraux et les secteurs pour tenir compte des différents besoins et priorités. Comme il est mentionné dans ce cadre, les gouvernements fédéraux, provinciaux et territoriaux définiront des cibles et des indicateurs pour le Canada qui leur permettront de mesurer leurs progrès et orienter leurs priorités respectives relatives aux piliers du cadre.

\author{
Affiliation \\ ${ }^{1}$ Agence de la santé publique du \\ Canada, Ottawa (Ontario)
}

*Correspondance: ccdic-clmti@ phac-aspc.gc.ca

Citation proposée : Centre de la lutte contre les maladies transmissibles et les infections. Résumé du cadre pancanadien sur les infections transmissibles sexuellement et par le sang. Relevé des maladies transmissibles au Canada 2018;44(7/8):201-4. https://doi.org/10.14745/ccdr.v44i78a05f

Mots-clés : infections transmissibles sexuellement et par le sang, cadre, Canada

\section{Introduction}

Les infections transmissibles sexuellement et par le sang (ITSS) restent un problème de santé important au Canada, même si elles peuvent être en grande partie évitées, traitées et, dans bien des cas, guéries. Les taux de certaines ITSS sont toujours à la hausse au Canada, et la dynamique mondiale visant à éliminer les nouvelles infections augmente. À cette fin, le Canada appuie les objectifs de développement durable des Nations Unies, ainsi que les stratégies mondiales du secteur de la santé pour lutter contre le $\mathrm{VIH}$, I'hépatite virale et les infections transmissibles sexuellement (ITS) du Programme commun des Nations Unies sur le VIH/SIDA (ONUSIDA) et de I'Organisation mondiale de la Santé (OMS). Ces objectifs et stratégies appellent les pays à prendre des mesures pour éliminer les ITSS en tant que problèmes majeurs de santé d'ici 2030 (1).

Pour orienter les efforts du Canada et contribuer aux efforts mondiaux visant à réduire leurs répercussions sur la santé, un cadre pancanadien, intitulé Réduction des répercussions sur la santé des infections transmissibles sexuellement et par le sang (ITSS) au Canada d'ici 2030 : un cadre d'action pancanadien sur les ITSS a été élaboré par l'Agence de la santé publique du Canada et a été appuyé par les ministres fédéral, provinciaux et territoriaux de la santé (2). Le présent article est un résumé du rapport complet.

\section{L'état des ITSS au Canada}

Au cours des dernières années, le nombre de nouveaux cas d'infection au VIH et au virus de l'hépatite $\mathrm{C}(\mathrm{VHC})$ est demeuré relativement stable au Canada, bien qu'il y ait des différences à l'échelle régionale et dans certaines communautés. On estime que 65000 personnes vivaient avec le VIH au Canada à la fin de l'année 2014, dont environ 20 \% n'étaient pas au courant de leur état (2). Les homosexuels, les bisexuels et les hommes ayant des relations sexuelles avec d'autres hommes (âgés de 15 ans et plus) représentaient environ $2,5 \%$ de la population, mais représentent pourtant près de $50 \%$ des personnes vivant avec une infection au $\mathrm{VIH}$ et plus de $50 \%$ des nouveaux cas d'infection au VIH au Canada en 2014 (3). En 2011, on estimait 
qu'entre 221000 et 246000 Canadiens étaient atteints d'une infection chronique par VHC, dont environ $44 \%$ n'étaient pas au courant de leur état (4).

Le nombre de nouveaux cas d'infection à Chlamydia trachomatis, d'infection gonococcique et de syphilis diagnostiqués augmente de façon constante depuis le milieu des années 1990, en dépit de nombreuses interventions de santé publique visant à prévenir, à diagnostiquer et à traiter ces infections (5). Entre 2005 et 2014, le taux de cas déclarés d'infection à Chlamydia trachomatis a augmenté de $49 \%$, le taux de cas déclarés d'infection gonococcique a augmenté de $61 \%$, et le taux de cas déclarés de syphilis a augmenté de $95 \%$.

Le Canada a réalisé des progrès dans des domaines tels que l'amélioration de l'accès à des traitements novateurs, la création de solides systèmes de surveillance pour le contrôle des infections et le renforcement de la capacité des organisations communautaires. Cependant, malgré les outils créés et les progrès réalisés, le nombre de nouvelles infections au Canada demeure à un niveau inacceptable. Il est temps pour le Canada de mettre de l'avant une nouvelle approche pour réduire le nombre de nouvelles infections, améliorer les résultats en matière de santé et contribuer aux efforts mondiaux.

\section{Une approche intégrée}

En 2016 et 2017, des mesures concrètes qui pourraient avoir d'importantes répercussions sur les taux d'ITSS au Canada ont été déterminées grâce à des sondages en ligne et à des réunions en personne avec un large éventail de partenaires et d'intervenants, notamment des personnes vivant avec le $\mathrm{VIH}$ et l'hépatite virale, des représentants des populations clés et des communautés et des organismes des Premières Nations, des Inuits et des Métis, des cliniciens et d'autres professionnels de la santé, des organismes communautaires et de la société civile, des chercheurs, des gouvernements des provinces et des territoires et des représentants de l'industrie pharmaceutique. Ces consultations ont permis d'éclairer le cadre au moyen d'un processus itératif.

Le cadre présente une approche globale et complète pour lutter contre les ITSS. Étant donné que les ITSS ont en commun des facteurs de risques courants, des comportements à risque, des voies de transmission ainsi que les populations clés qui sont touchées par elles, le Canada s'est orienté vers une approche intégrée. En même temps, il est reconnu que les approches propres aux infections demeurent adaptées dans certaines circonstances ou communautés. On s'attend à ce que les partenaires et les intervenants à l'échelle du Canada consultent le cadre pour éclairer leur travail. Une approche collaborative sera essentielle à la réussite, puisqu'aucun secteur ne peut réduire seul les répercussions des ITSS sur la santé.

\section{Cadre pancanadien sur les ITSS}

Le cadre établit une vision pour le Canada dans laquelle les ITSS sont rares et où les personnes vivant avec les ITSS reçoivent les soins et le soutien dont elles ont besoin. Le succès du cadre sera mesuré par rapport aux objectifs en matière d'ITSS à l'échelle mondiale, et les progrès réalisés dans l'atteinte de ces objectifs appuieront les objectifs stratégiques suivants, plus généraux, pour le Canada :
- Réduire l'incidence des ITSS au Canada;

- Améliorer l'accès au dépistage, au traitement, aux soins de santé et au soutien continus;

- Réduire la stigmatisation et la discrimination qui créent une vulnérabilité aux ITSS.

Une série de principes directeurs ont été adoptés pour guider la façon dont nous luttons collectivement contre les ITSS au Canada (tableau 1).

\section{Tableau 1 : Principes directeurs du cadre pancanadien de 2018 pour réduire les ITSS (2)}

\begin{tabular}{|c|c|}
\hline $\begin{array}{l}\text { Principes } \\
\text { directeurs }\end{array}$ & Définitions \\
\hline $\begin{array}{l}\text { Participation } \\
\text { significative des } \\
\text { personnes vivant } \\
\text { avec le VIH et } \\
\text { I'hépatite virale et des } \\
\text { populations clés }\end{array}$ & $\begin{array}{l}\text { Les personnes vivant avec le VIH et l'hépatite } \\
\text { virale et les populations clés participent de } \\
\text { façon significative à l'élaboration et à la mise en } \\
\text { œuvre des politiques et des programmes qui les } \\
\text { concernent. }\end{array}$ \\
\hline $\begin{array}{l}\text { Progression vers } \\
\text { la verité et la } \\
\text { réconciliation }\end{array}$ & $\begin{array}{l}\text { Des politiques et des programmes pour lutter } \\
\text { contre les ITSS chez les peuples autochtones } \\
\text { sont élaborés par et avec les communautés } \\
\text { des Premières Nations, des Inuits et des Métis, } \\
\text { dans une relation qui repose sur le respect } \\
\text { mutuel; ils sont fondés sur la compréhension et } \\
\text { la reconnaissance des effets permanents de la } \\
\text { colonisation, des conséquences sociales et sur } \\
\text { la santé des pensionnats indiens, des inégalités } \\
\text { structurelles et du racisme systémique, de même } \\
\text { que sur la réponse à ces enjeux. }\end{array}$ \\
\hline Approche intégrée & $\begin{array}{l}\text { Les interventions et les programmes visent à } \\
\text { prendre en compte la complexité et la nature } \\
\text { interdépendante des facteurs de risque et des } \\
\text { voies de transmission des ITSS, tout en permettant } \\
\text { l'adoption d'approches propres aux maladies } \\
\text { lorsqu'elles sont pertinentes. }\end{array}$ \\
\hline Pertinence culturelle & $\begin{array}{l}\text { Les politiques et les programmes de lutte contre } \\
\text { les ITSS reflètent et respectent les réalités et les } \\
\text { pratiques culturelles tout en assurant la sécurité } \\
\text { des personnes et des collectivités. }\end{array}$ \\
\hline Droits de la personne & $\begin{array}{l}\text { Chaque personne, sans égard à son orientation } \\
\text { sexuelle, à sa race, à sa culture, à son genre, à } \\
\text { ses capacités ou à ses pratiques personnelles, est } \\
\text { importante, et ses droits sont reconnus, respectés } \\
\text { et promus. }\end{array}$ \\
\hline $\begin{array}{l}\text { Équité en matière de } \\
\text { santé }\end{array}$ & $\begin{array}{l}\text { Chaque personne, sans égard à son sexe, son } \\
\text { genre, sa race, son revenu, son orientation } \\
\text { sexuelle, son emplacement géographique, son } \\
\text { statut, son âge ou sa culture, a un accès équitable } \\
\text { à des renseignements et à des services de qualité } \\
\text { fournis par des professionnels de la santé et } \\
\text { d'autres fournisseurs de première ligne qualifiés. }\end{array}$ \\
\hline $\begin{array}{l}\text { Approche } \\
\text { multisectorielle }\end{array}$ & $\begin{array}{l}\text { Des approches multisectorielles et } \\
\text { multidisciplinaires de prévention et de soins } \\
\text { sont adoptées pour améliorer la collaboration et } \\
\text { s'assurer que les interventions tiennent compte de } \\
\text { l'ensemble des besoins de la personne en matière } \\
\text { de mieux-être. }\end{array}$ \\
\hline $\begin{array}{l}\text { Politiques et } \\
\text { programmes fondés sur } \\
\text { des données probantes }\end{array}$ & $\begin{array}{l}\text { Les interventions et les programmes sont } \\
\text { systématiquement élaborés et orientés par les } \\
\text { données de surveillance, les recherches ainsi que } \\
\text { d'autres données probantes récentes. }\end{array}$ \\
\hline
\end{tabular}

Le cadre comprend quatre piliers interconnectés qui s'étendent au suivi des soins des ITSS dans son ensemble :

- La prévention fait la promotion des connaissances sur la santé sexuelle, change les attitudes et les comportements et appuie les interventions existantes ou émergentes en matière de prévention (p. ex., la prophylaxie préexposition contre le VIH et les vaccins);

- Le dépistage fait la promotion de l'offre systématique de tests de dépistage et de nouvelles analyses au point de service, et lutte pour éliminer les obstacles existants à l'accès aux tests de dépistage; 
- L'amorce des soins et des traitements fait la promotion de soins et de traitement en temps opportun ainsi que de la prise de nouveaux médicaments pour toutes les populations;

- Les soins et le soutien continus font la promotion des efforts visant à maintenir et à renouer le dialogue avec les personnes atteintes d'ITSS chroniques au sein des services de soins et comprennent l'élaboration de modèles de soins " globaux » interdisciplinaires et multisectoriels qui intègrent les services liés aux ITSS dans les cliniques de soins primaires.

En plus de ses principaux piliers, le cadre reconnaît la nécessité de créer un environnement favorable pour assurer la réussite des mesures liées aux piliers du cadre. Un environnement favorable crée les conditions nécessaires pour assurer une couverture équitable, augmenter l'adoption des services de santé et en améliorer la qualité. Cet environnement englobe l'ensemble de conditions juridiques, sociales, culturelles, physiques et structurelles interreliées qui contribuent au succès des programmes, des politiques et des mesures liées aux ITSS. La figure 1 présente un schéma du cadre.

Figure 1 : Un cadre pancanadien pour réduire les répercussions sur la santé des ITSS au Canada

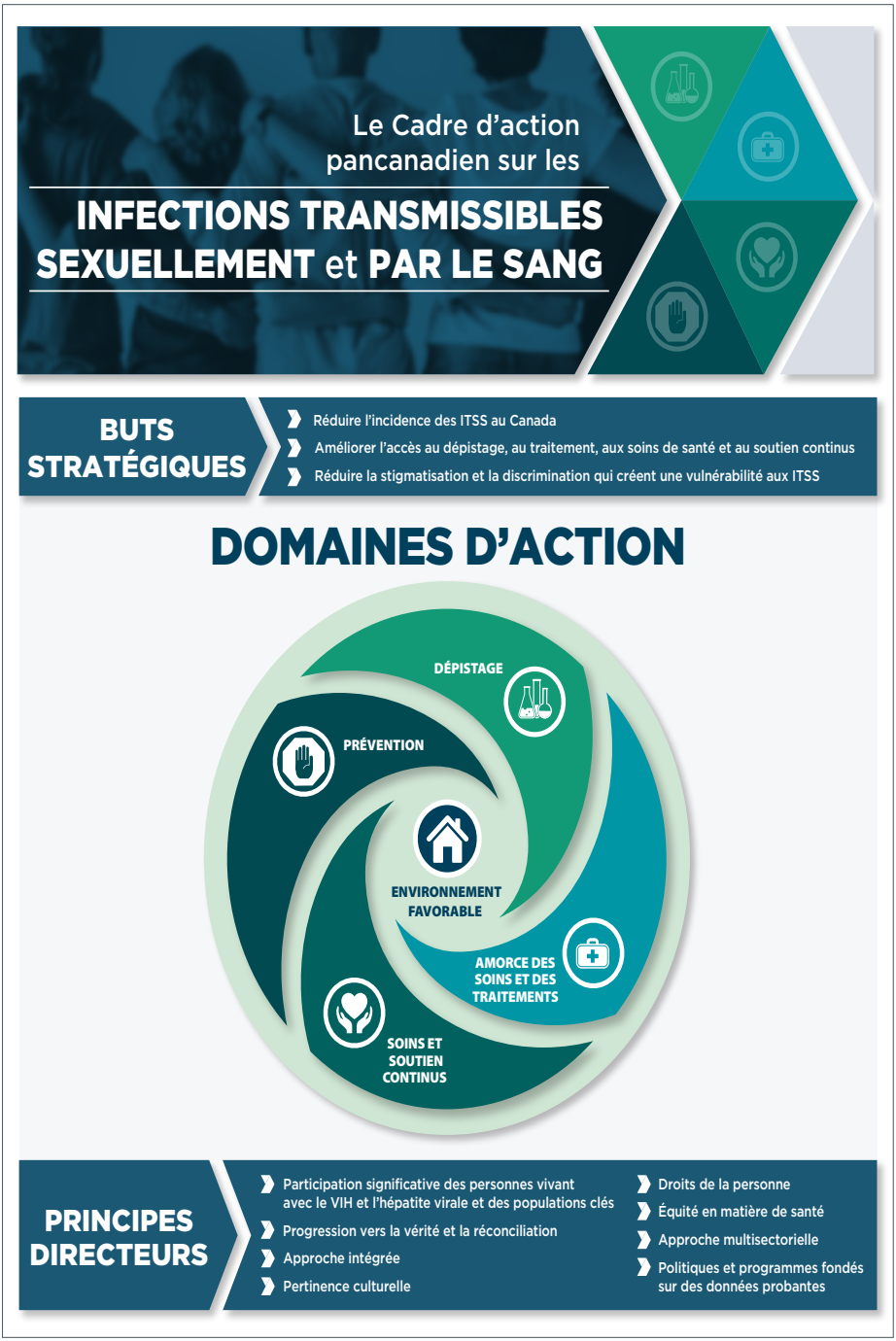

Le cadre définit 26 possibilités d'action réparties entre tous les piliers, y compris l'environnement favorable qui peut être utilisé pour orienter les mesures prises par des gens de populations clés tels que les dirigeants et les communautés autochtones, la société civile, les professionnels de la santé, les associations professionnelles et les organismes d'attribution, le secteur privé et de tous les niveaux de gouvernement. Enfin, le cadre reconnaît également les systèmes de surveillance, la recherche, la mobilisation des connaissances et la surveillance et l'évaluation comme d'importantes activités transversales.

\section{Conclusion et prochaines étapes}

Grâce au cadre pancanadien, le Canada unifie et communique une approche commune pour lutter contre les ITSS tout en respectant la souplesse exigée par les administrations et les secteurs pour tenir compte des différents besoins et priorités. Les gouvernements fédéral, provinciaux et territoriaux se sont engagés à développer des cibles et des indicateurs pour le Canada qui leur permettront de mesurer leurs progrès et $d$ 'orienter leurs priorités respectives relatives aux piliers du cadre.

Le gouvernement du Canada élabore un plan de mise en œuvre qui déterminera des mesures précises que le gouvernement fédéral devra prendre au cours des cinq prochaines années et qui contribueront à l'élimination des ITSS et à l'atteinte des objectifs stratégiques établis dans le cadre.

\section{Remerciements}

L'Agence de la santé publique du Canada souhaite remercier toutes les personnes qui ont contribué de leur temps et de leur expertise à la préparation du rapport Réduction des répercussions sur la santé des infections transmissibles sexuellement et par le sang au Canada d'ici 2030 : un cadre d'action pancanadien.

\section{Références}

1. Organisation mondiale de la Santé. Stratégie mondiale du secteur de la santé contre le VIH, 2016-2021. Geneva: OMS; 2016. http://apps.who.int/iris/bitstream/10665/250576/1/ WHO-HIV-2016.05-fre.pdf?ua=1

2. Réduction des répercussions sur la santé des infections transmissibles sexuellement et par le sang au Canada d'ici 2030 : un cadre d'action pancanadien sur les ITSS.Ottawa (ON): ASPC; 2018. https://www.canada. $\mathrm{ca} / \mathrm{fr} /$ sante-publique/services/maladies-infectieuses/ sante-sexuelle-infections-transmissibles-sexuellement/ rapports-publications/infections-transmissible s-sexuellement-sang-cadre-action.html

3. Agence de la santé publique du Canada. Résumé : Mesurer les progrès réalisés par le Canada en ce qui concerne les cibles 90-90-90 pour le VIH. Ottawa (ON): ASPC; 2016. https://www.canada.ca/fr/sante-publique/services/ publications/maladies-et-affections/resume-mesure r-les-progres-realises-par-le-canada-cibles-90-90-90-pour-levih.html

4. Agence de la Santé Publique du Canada. Résumé : Estimations de l'incidence de la prévalence, et de la 
proportion non diagnostiquée au VIH au Canada, 2014.

Ottawa (ON): ASPC;2015. https://www.canada.ca/fr/santepublique/services/publications/maladies-et-affections/ resume-estimations-incidence-prevalence-et-proportion-nondiagnostiquee-vih-canada-2014.html

5. Trubnikov M, Yan P, Archibald C. Estimation de la prévalence de l'infection par le virus de l'hépatite C au Canada, 2011. Relevé des maladies transmissibles au Canada 2014
Dec;40(19):429-36. PubMed (https://www.ncbi.nlm.nih.gov/ pubmed/29769874)

6. Agence de la santé publique du Canada. Rapport sur les infections transmissibles sexuellement au Canada : 2013-2014. Ottawa: ASPC; 2017. https://www.canada.

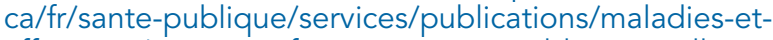
affections/rapport-infections-transmissibles-sexuellementcanada-2013-14.html 